\title{
Untargeted Chemometrics Evaluation of the Effect of Processing Technique on Phytochemical Profiles and Antioxidant Activities in Common Vegetables
}

Junyi Wang, ${ }^{\mathrm{a}, \mathrm{b}}$ G.K. Jayaprakasha, ${ }^{\mathrm{a}^{*}}$ and Bhimanagouda S.Patil ${ }^{\mathrm{a}, \mathrm{b}^{*}}$

${ }^{a}$ Vegetable and Fruit Improvement Center, Department of Horticultural Sciences, ${ }^{b}$ Department of Nutrition and Food Science, Texas A\&M University, 1500 Research Parkway, Suite A120, College Station, TX 77845, USA

*Corresponding authors

G.K. Jayaprakasha

Tel.: +1 979845 2743; fax: +1 9798624522

E-mail: gkjp@tamu.edu

Bhimanagouda S. Patil,

Tel.: +1 979862 4521; fax: +1 9798624522

E-mail: b-patil@tamu.edu 

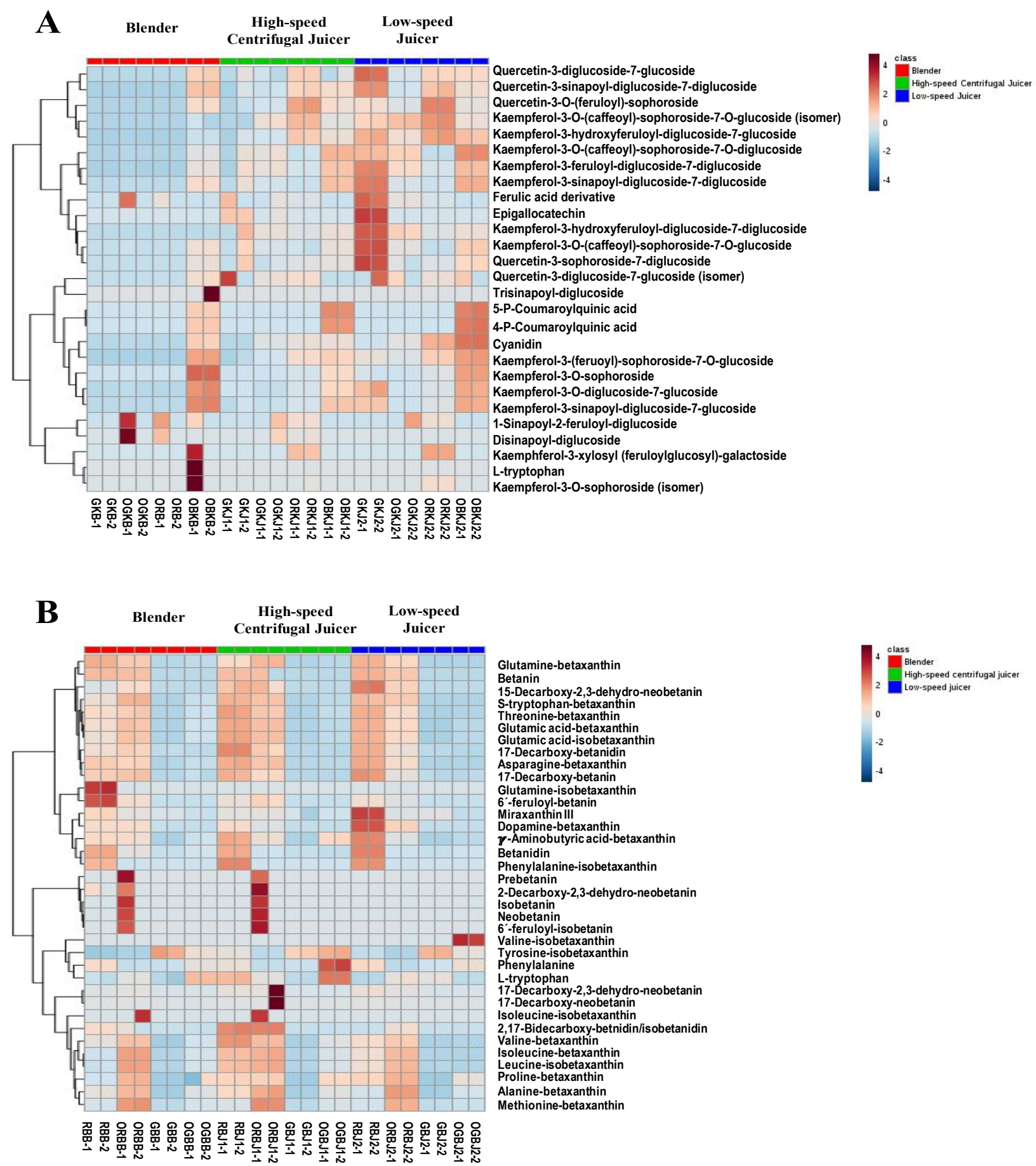

Figure S1. Relative abundance of significantly changed metabolites are presented as box-and-whisker plot in (A) kale and (B) beet processed samples. The color box for each compound indicates the abundance of the compound, with the brown color annotates higher and the blue color represents lower abundance. The $x$ and $y$ axis represent sample type and compounds names, respectively. Sample types were shown as vegetable name plus processing techniques. Processing techniques were abbreviated as B/J1/J2 representing blending/high-speed centrifugal juicing/low-speed juicing. Kale sample name abbreviations: GK: green kale; OGK: organic green kale; OBK, 
organic black kale. Beet sample name abbreviations: RB: red beet; ORB: organic red beet; GB: golden beet; OGB: organic golden beet. 
Table S1. Commercial name, scientific name, yield, and color measurement of 21 vegetables processed by three juicing techniques.

\begin{tabular}{|c|c|c|c|c|c|c|}
\hline $\begin{array}{l}\text { Scientific } \\
\text { name }\end{array}$ & Name (color) & Processing & Yield (\%) & $\mathrm{L}^{*}$ & $\mathrm{a}^{*}$ & $\mathrm{~b}^{*}$ \\
\hline \multirow{10}{*}{ 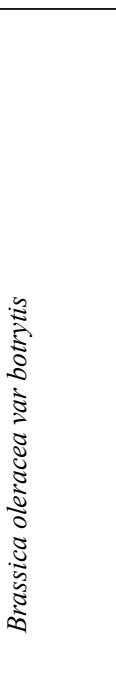 } & Cauliflower (W) & Oster Blender & 93.1 & $73.62 \pm 0.40$ & $0.23 \pm 0.40$ & $26.16 \pm 0.36$ \\
\hline & Cauliflower (W) & Breville Juice Extractor & 35.1 & $77.08 \pm 0.39$ & $-5.68 \pm 0.11$ & $25.32 \pm 0.16$ \\
\hline & Cauliflower (W) & Omega Juicer & 35.6 & $80.15 \pm 0.71$ & $-3.17 \pm 0.04$ & $19.76 \pm 0.55$ \\
\hline & Cauliflower (Y) & Oster Blender & 88.2 & $71.61 \pm 0.38$ & $8.13 \pm 0.11$ & $47.17 \pm 0.49$ \\
\hline & Cauliflower (Y) & Breville Juice Extractor & 27.8 & $74.11 \pm 0.78$ & $5.99 \pm 0.38$ & $46.92 \pm 0.81$ \\
\hline & Cauliflower (Y) & Omega Juicer & 29.6 & $70.31 \pm 0.65$ & $6.11 \pm 0.88$ & $49.02 \pm 1.38$ \\
\hline & Cauliflower (G) & Oster Blender & 92.1 & $60.49 \pm 0.40$ & $-9.04 \pm 0.01$ & $31.72 \pm 0.80$ \\
\hline & Cauliflower (G) & Breville Juice Extractor & 29.8 & $74.93 \pm 0.22$ & $12.79 \pm 0.10$ & $39.95 \pm 0.48$ \\
\hline & Cauliflower (G) & Omega Juicer & 31.2 & $74.03 \pm 0.02$ & $10.01 \pm 0.10$ & $31.23 \pm 0.32$ \\
\hline & Kale (G) & Oster Blender & 94.3 & $33.90 \pm 0.01$ & $-6.40 \pm 0.08$ & $12.38 \pm 0.19$ \\
\hline \multirow{17}{*}{ 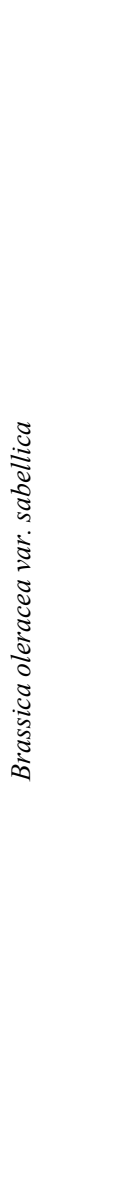 } & Kale (G) & Breville Juice Extractor & 17.6 & $45.81 \pm 0.95$ & $17.21 \pm 0.95$ & $28.54 \pm 2.38$ \\
\hline & Kale (G) & Omega Juicer & 36.8 & $36.41 \pm 0.27$ & $10.31 \pm 0.08$ & $11.70 \pm 0.16$ \\
\hline & Kale (GO) & Oster Blender & 92.3 & $30.43 \pm 0.34$ & $-3.82 \pm 0.05$ & $4.23 \pm 0.06$ \\
\hline & Kale (GO) & Breville Juice Extractor & 33.7 & $32.57 \pm 0.17$ & $-6.53 \pm 0.14$ & $7.98 \pm 0.28$ \\
\hline & Kale (GO) & Omega Juicer & 42.8 & $31.14 \pm 0.37$ & $-6.53 \pm 0.12$ & $7.22 \pm 0.27$ \\
\hline & Kale (RO) & Oster Blender & 92.7 & $30.78 \pm 0.34$ & $-2.87 \pm 0.13$ & $5.99 \pm 0.53$ \\
\hline & Kale (RO) & Breville Juice Extractor & 35.9 & $29.16 \pm 0.77$ & $-1.39 \pm 0.89$ & $3.06 \pm 1.15$ \\
\hline & Kale (RO) & Omega Juicer & 55.0 & $28.87 \pm 0.82$ & $-0.65 \pm 0.33$ & $1.43 \pm 0.28$ \\
\hline & Kale (BO) & Oster Blender & 94.6 & $29.27 \pm 0.18$ & $-4.86 \pm 0.05$ & $5.01 \pm 0.59$ \\
\hline & Kale (BO) & Breville Juice Extractor & 27.2 & $30.57 \pm 0.08$ & $-6.22 \pm 0.07$ & $5.49 \pm 0.12$ \\
\hline & Kale (BO) & Omega Juicer & 52.3 & $29.41 \pm 0.64$ & $-5.38 \pm 0.38$ & $5.47 \pm 0.56$ \\
\hline & Turnip (P) & Oster Blender & 93.1 & $59.7 \pm 0.08$ & $9.35 \pm 0.12$ & $21.49 \pm 0.14$ \\
\hline & Turnip (P) & Breville Juice Extractor & 44.9 & $78.74 \pm 1.13$ & $0.28 \pm 0.22$ & $11.52 \pm 1.38$ \\
\hline & Turnip (P) & Omega Juicer & 48.6 & $77.81 \pm 0.38$ & $0.34 \pm 0.18$ & $9.84 \pm 1.19$ \\
\hline & Turnip (W) & Oster Blender & 79.4 & $68.44 \pm 0.31$ & $2.78 \pm 0.04$ & $25.99 \pm 0.15$ \\
\hline & Turnip (W) & Breville Juice Extractor & 52.1 & $79.45 \pm 0.88$ & $-2.17 \pm 0.07$ & $12.67 \pm 1.00$ \\
\hline & Turnip (W) & Omega Juicer & 47.0 & $82.85 \pm 1.02$ & $-0.77 \pm 0.08$ & $8.65 \pm 0.95$ \\
\hline
\end{tabular}




\begin{tabular}{|c|c|c|c|c|c|c|}
\hline & Radish (R) & Oster Blender & 92.0 & $54.30 \pm 0.53$ & $17.81 \pm 0.48$ & $19.35 \pm 0.32$ \\
\hline & Radish (R) & Breville Juice Extractor & 50.6 & $66.96 \pm 2.52$ & $15.69 \pm 1.04$ & $5.78 \pm 0.21$ \\
\hline & Radish (R) & Omega Juicer & 53.3 & $80.19 \pm 0.24$ & $9.54 \pm 0.38$ & $2.63 \pm 0.09$ \\
\hline & Radish (RO) & Oster Blender & 93.6 & $50.28 \pm 1.33$ & $31.13 \pm 0.98$ & $7.28 \pm 0.76$ \\
\hline & Radish (RO) & Breville Juice Extractor & 52.9 & $63.24 \pm 1.05$ & $20.08 \pm 0.35$ & $4.67 \pm 0.19$ \\
\hline & Radish (RO) & Omega Juicer & 56.6 & $67.36 \pm 2.17$ & $15.86 \pm 1.63$ & $3.97 \pm 0.71$ \\
\hline 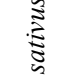 & Radish (G) & Oster Blender & 85.8 & $35.53 \pm 0.07$ & $25.77 \pm 0.17$ & $8.43 \pm 0.09$ \\
\hline \begin{tabular}{l}
$\dot{0}$ \\
\multirow{3}{*}{} \\
\end{tabular} & Radish (G) & Breville Juice Extractor & 50.1 & $47.81 \pm 2.53$ & $43.74 \pm 1.44$ & $4.44 \pm 0.77$ \\
\hline 离 & Radish (G) & Omega Juicer & 51.5 & $46.55 \pm 0.52$ & $42.84 \pm 0.61$ & $4.05 \pm 0.46$ \\
\hline$\Xi$ & Radish (W) & Oster Blender & 87.7 & $72.57 \pm 0.72$ & $-1.74 \pm 0.01$ & $17.17 \pm 0.27$ \\
\hline$\lesssim$ & Radish (W) & Breville Juice Extractor & 56.0 & $84.14 \pm 1.58$ & $-0.42 \pm 0.06$ & $3.85 \pm 0.15$ \\
\hline 竧 & Radish (W) & Omega Juicer & 63.2 & $85.46 \pm 0.77$ & $-0.17 \pm 0.09$ & $3.53 \pm 0.71$ \\
\hline & Beet $(\mathrm{R})$ & Oster Blender & 92.0 & $52.55 \pm 0.21$ & $7.74 \pm 0.16$ & $29.46 \pm 0.05$ \\
\hline & Beet $(\mathrm{R})$ & Breville Juice Extractor & 45.4 & $71.31 \pm 0.72$ & $-4.11 \pm 0.52$ & $44.58 \pm 1.20$ \\
\hline & Beet $(\mathrm{R})$ & Omega Juicer & 53.7 & $75.81 \pm 0.58$ & $-5.90 \pm 0.14$ & $38.72 \pm 0.87$ \\
\hline & Beet (RO) & Oster Blender & 93.6 & $48.40 \pm 0.45$ & $21.92 \pm 0.27$ & $31.85 \pm 0.33$ \\
\hline & Beet (RO) & Breville Juice Extractor & 37.6 & $60.45 \pm 0.82$ & $32.03 \pm 0.94$ & $47.07 \pm 1.98$ \\
\hline & Beet (RO) & Omega Juicer & 39.4 & $62.00 \pm 0.72$ & $29.94 \pm 1.18$ & $50.01 \pm 1.06$ \\
\hline & Beet (G) & Oster Blender & 91.4 & $32.47 \pm 0.18$ & $2.03 \pm 0.01$ & $12.72 \pm 0.09$ \\
\hline & Beet $(\mathrm{G})$ & Breville Juice Extractor & 37.8 & $34.25 \pm 1.16$ & $6.72 \pm 0.63$ & $17.11 \pm 1.77$ \\
\hline & Beet $(\mathrm{G})$ & Omega Juicer & 44.2 & $31.86 \pm 0.39$ & $6.07 \pm 0.12$ & $12.78 \pm 0.46$ \\
\hline & Beet (GO) & Oster Blender & 93.6 & $28.10 \pm 0.07$ & $1.41 \pm 0.01$ & $8.79 \pm 0.07$ \\
\hline$\frac{5}{5}$ & Beet (GO) & Breville Juice Extractor & 45.4 & $38.46 \pm 0.36$ & $9.21 \pm 0.31$ & $21.43 \pm 1.30$ \\
\hline$\stackrel{\Xi}{\square}$ & Beet (GO) & Omega Juicer & 45.3 & $57.80 \pm 0.31$ & $10.07 \pm 0.57$ & $56.53 \pm 0.34$ \\
\hline & Baby Carrot (O) & Oster Blender & 68.3 & $48.40 \pm 0.45$ & $21.92 \pm 0.27$ & $31.85 \pm 0.33$ \\
\hline : & Baby Carrot (O) & Breville Juice Extractor & 39.2 & $60.45 \pm 0.82$ & $32.03 \pm 0.94$ & $47.07 \pm 1.98$ \\
\hline 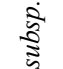 & Baby Carrot (O) & Omega Juicer & 44.3 & $62.00 \pm 0.72$ & $29.94 \pm 1.18$ & $50.01 \pm 1.06$ \\
\hline 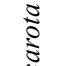 & Baby Carrot (P) & Oster Blender & 88.8 & $29.20 \pm 0.07$ & $5.19 \pm 0.16$ & $2.59 \pm 0.14$ \\
\hline ב్ & Baby Carrot (P) & Breville Juice Extractor & 38.1 & $25.75 \pm 0.98$ & $6.45 \pm 0.17$ & $-2.20 \pm 0.34$ \\
\hline & Baby Carrot (P) & Omega Juicer & 37.9 & $24.83 \pm 1.40$ & $5.42 \pm 0.63$ & $-2.10 \pm 0.17$ \\
\hline
\end{tabular}




\begin{tabular}{|c|c|c|c|c|c|}
\hline Baby Carrot (Y) & Oster Blender & 76.9 & $46.09 \pm 0.32$ & $8.95 \pm 0.14$ & $30.45 \pm 0.28$ \\
\hline Baby Carrot (Y) & Breville Juice Extractor & 43.9 & $62.44 \pm 1.66$ & $9.32 \pm 0.34$ & $52.19 \pm 0.24$ \\
\hline Baby Carrot (Y) & Omega Juicer & 33.7 & $59.62 \pm 0.67$ & $10.47 \pm 0.31$ & $51.79 \pm 1.27$ \\
\hline Baby Carrot (W) & Oster Blender & 91.5 & $52.55 \pm 0.21$ & $7.74 \pm 0.16$ & $29.46 \pm 0.05$ \\
\hline Baby Carrot (W) & Breville Juice Extractor & 52.0 & $71.31 \pm 0.72$ & $-4.11 \pm 0.52$ & $44.58 \pm 1.20$ \\
\hline Baby Carrot (W) & Omega Juicer & 40.4 & $75.81 \pm 0.58$ & $-5.90 \pm 0.14$ & $38.72 \pm 0.87$ \\
\hline
\end{tabular}

Color: W: white, Y: yellow, G: green, GO: green organic, BO: black organic, P: purple, WB: white baby, R: red, RO: red organic; G: golden, GO: golden organic. L*: brightness; $a^{*}$ : redness; b*yellowness; $C^{*}$ : Chroma; h: hue angle. 